\title{
Cellular and Subcellular Modifications Induced by Perinatal Asphyxia. High Resolution 3-d Reconstruction Study
}

Francisco Capani and Rodolfo Kolliker Frers

Instituto de Investigaciones Cardiológicas (ININCA), UBA-CONICET, Buenos Aires, Buenos Aires, Argentina

Statement of the Problem: Diminish in the oxygen levels prompted short and long-term alterations in synapses and related structures that are related to neuronal dysfunction and death. Perinatal asphyxia (PA) is an obstetric complication produced by an impaired gas exchange that lead to neonatal mortality and is a determinant factor for neurodevelopmental disorders. Since pathophysiological mechanisms triggered by PA are not still totally unveiled, we investigated the changes in the cytoskeleton organization, synapse and astrocyts in the nervous tissue.

Methodology \& Theoretical Orientation: For this study, we used a well-established murine model of PA [1]. After one, 2, 4 and 6 months of severe PA (20 min) rats were sacrificed and their brains were analyzed by combining photooxidation, conventional electron microscopy, and 3-D reconstruction techniques [1]. Findings: After one month of PA, we found an increase in the F-actin staining in neostriatal and hippocampal dendritic spines together with some filopodia-likes structures, a typical embryonic type of spines in photooxidated tissue [2] [Fig 1 A). In contrast, after second month of PA, spines were less consistent stained. In addition, we observed an increment of marker for neuronal and glial dysfunction such as GFAP, neurofilament and MAP-2 [4,5]. These modifications were more striking defined after 4 months of PA [3,4]. After 6 months of PA postsynaptic densities (PSDs) in neostriatum were highly modified. Using three-D reconstructions and electron tomography we were able to find clear signs of degeneration in the asphyctic PSDs (Fig 1 B and C) [1]

Conclusion \& Significance: Therefore, we hypothesize that the cytoskeletal changes induced by PA in the rat CNS could lead to the dramatic modifications in synapse and related structures that trigger neuronal damage. In addition, electron tomography, 3-D reconstruction and photooxidation contributed to dissect critical alterations generated by PA that are not easily displayed using conventional microscopic techniques. These findings migth contribute to generate new therapeutic tools in a is lacked of them 
A

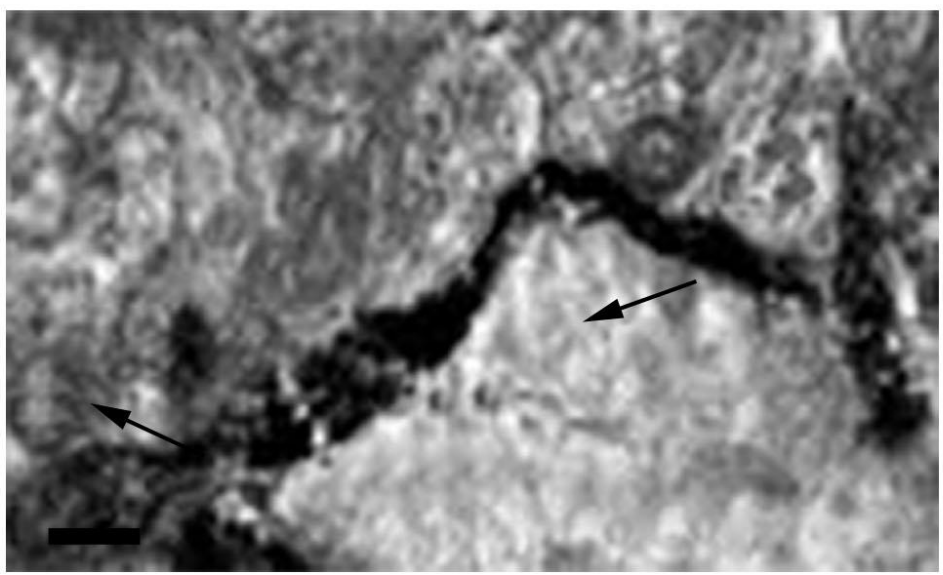

B
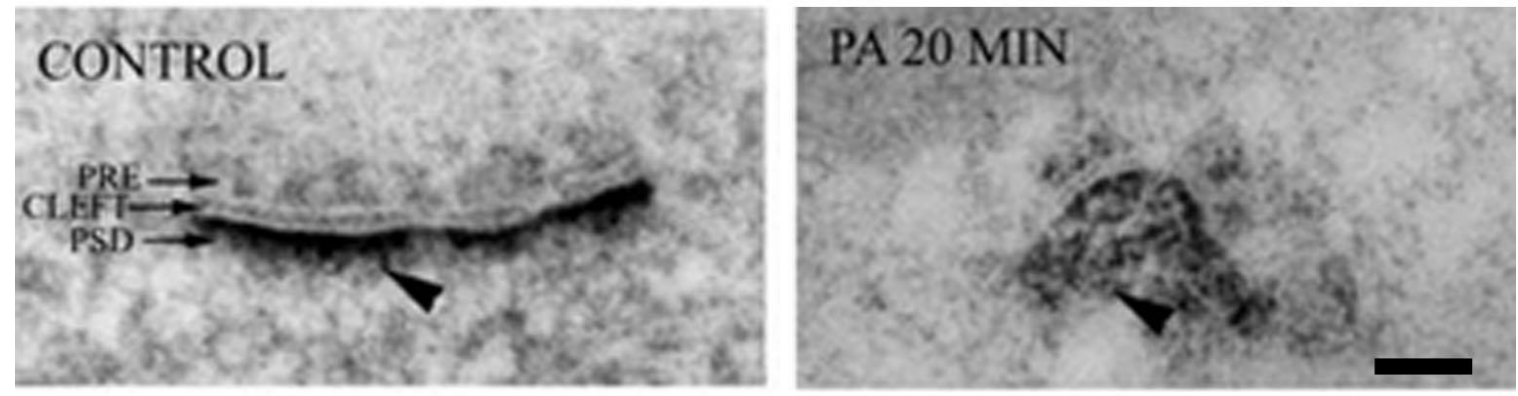

C
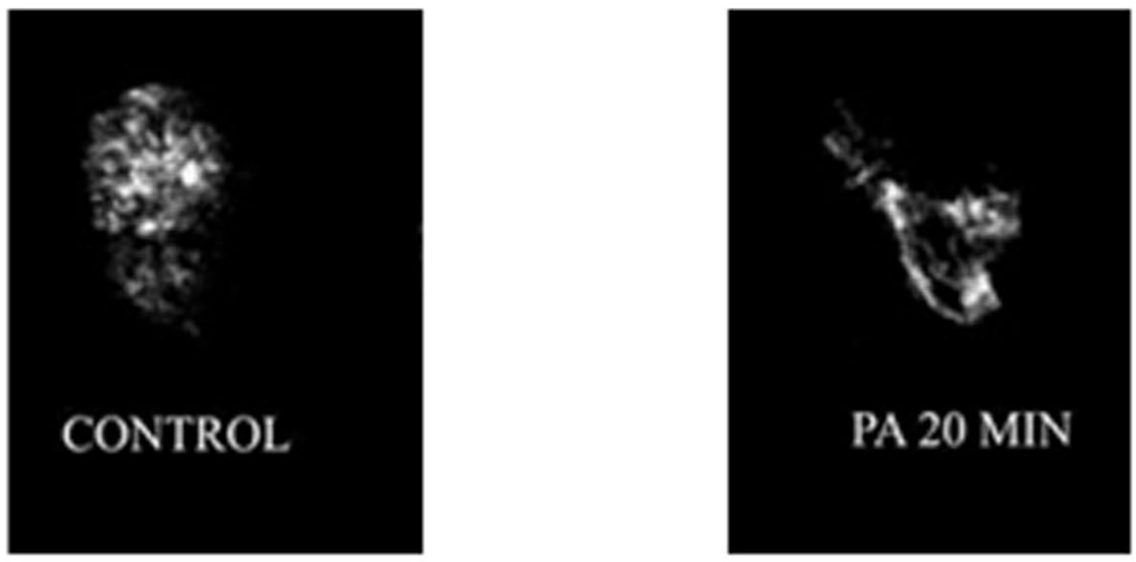

Figure 1. A) Electron micrograph of filopodia F-actin stained in hippocampus PA rat. The magnification of a filopodium allows us to observe its long structure and the parenteral dendrites (arrows). Scale bar: 1 $\mu \mathrm{m}$. (B) Electron micrograph 1 E-PTA staining in neostriatum. Severe PA post-synaptic density showed a 
clear increment in the thickness. Scale bar $0.5 \mu \mathrm{m}$ (C) Three-reconstruction of individual post-synaptic density. After 20 min of PA post-synaptic clear signs of degeneration were observed.

\section{References}

[1] Capani, F., Saraceno, G.E., Boti, V., Aón-Bertolino, L., Madureira de Oliveria, D., Barreto, G., Galeano, P., Giraldez-Alvarez, L.D., Coirini H. (2009): Protein ubiquitination in postsynaptic densities after hypoxia in rat neostriatum is blocked by hypothermia. Exp Neurol. 219, 404-13.

[2] Saraceno GE, Guelman LR, Castilla R, Udovin LD, Ellisman MH, Capani F (2016) Consequences of excessive plasticity in the hippocampus induced by perinatal asphyxia. Exp Neurol. 286:116-123.

[3] Saraceno GE, R, Barreto GE, Gonzalez J, Kölliker-Frers RA, Capani F (2012) Hippocampal dendritic spines modifications induced by perinatal asphyxia. Neural Plast. 2012:873532

[4] Muñiz, J, Romero, J, Holubiec, M, Barreto, G, González, J, Saint-Martin, M, Calvo, E, Carlos Cavicchia, J, Castilla, R, Capani, F. (2014) Neuroprotective effects of hypothermia on synaptic actin cytoskeletal changes induced by perinatal asphyxia. Brain Res. 14; 1563:81-90

5] Romero JI, Holubiec MI, Logica Tornatore T, Rivière S, Hanschmann EM, Kölliker-Frers RA, Tau J, Blanco E, Galeano P, Rodríguez de Fonseca F, Lillig CH, Capani F. Neuronal Damage Induced by Perinatal Asphyxia Is Attenuated by Postinjury Glutaredoxin-2 Administration. Oxidative Medicine and Cellular Longevity Oxid Med Cell Longev 2017:4162465 